\title{
Spatiotemporal Visualization of DNA Replication Dynamics
}

\author{
Marius Reinhart, Corella S. Casas-Delucchi, and M. Cristina Cardoso
}

\begin{abstract}
The ability of cells to copy their DNA allows them to transmit their genetic information to their progeny. In such, this central biological process preserves the instructions that direct the entire development of a cell. Earlier biochemical analysis in vitro and genetic analysis in yeast laid the basis of our understanding of the highly conserved mechanism of DNA replication. Recent advances on labeling and live-cell microscopy permit now the dissection of this fundamental process in vivo within the context of intact cells. In this chapter, we describe in detail how to perform multiple DNA replication labeling and detection allowing high spatial resolution imaging, as well as how to follow DNA replication in living cells allowing high temporal resolution imaging.
\end{abstract}

Key words DNA replication, Fluorescent protein, Immunofluorescence staining, Live-cell microscopy, Nucleotide pulse labeling

\section{Introduction}

The accurate duplication of the genome is the basis for cell proliferation. The process of DNA replication takes place during $S$-phase and is organized both spatially and temporally [1], so that the activation of single replication origins throughout $S$-phase results in conserved in situ labeling patterns that change as S-phase progresses [2-4]. Nevertheless, the mechanism by which active replication spreads along a chromosome remains unclear.

First hints about the organization of active replication sites were obtained by pioneering experiments using radioactively labeled nucleotides, which are incorporated during the DNA synthesis process. These studies provided very valuable data on the localization of active replication sites along single stretched DNA fibers and further presented the first in situ data on DNA replication in mammalian cells $[5,6]$. However, detailed spatial

Marius Reinhart and Corella S. Casas-Delucchi have contributed equally to this work. 
information about the in situ organization of replication sites had to wait for the development of the first specific antibodies against halogenated nucleotides in the 1980s $[2,7]$. In situ detection of incorporated nucleotides and later of replication factors $[8,9]$ provided quite detailed spatial information; however, they represent single snapshots of the dynamic replication process. The combination of two pulses with differently modified nucleotides, on the other hand, for the first time ascertained the organization of DNA replication in situ both spatially and temporally: each snapshot gives detailed spatial information and the correlation between both provides valuable information on the way active sites of DNA replication progress in situ $[10,11]$. Later, the sequential use of directly labeled nucleotides made it possible to visualize sites of DNA synthesis in living cells at different time points. However, fluorescent dUTPs are not permeable through the cell membrane and need to be delivered by microinjection of cells or scratch loading ([12] and references therein). However, these elegant methods have the drawback that it is technically difficult to get even one nucleotide into the cell and incorporated into DNA making it not widely used and difficult to extend to multiple labelings. Finally, the development of fluorescent proteins for cell biological applications [13] made the next step possible, namely, the visualization of DNA replication progression in vivo over longer periods of time [14, 15]. Following fluorescently tagged PCNA in vivo, Sporbert et al. showed that new replication foci are always activated adjacently to already active ones [16] and proposed a domino model to explain the propagation of active DNA replication, where active replication results in its own propagation by, for instance, destabilizing chromatin/DNA and thereby facilitating firing of nearby origins [17].

Here, we present in detail two very useful methods, both allowing visualization of DNA replication dynamics in situ using fluorescence microscopy. The first, consisting of time-lapse imaging of living cells expressing fluorescently tagged replication proteins, provides both 3D spatial information and especially extensive temporal information. The second, a further development of pulse and chase experiments using modified nucleotides to label replication in situ [18], allows the visualization of up to four combined snapshots of replication sites active at selected time intervals. While the former approach can be used to visualize replication progression continuously, the latter facilitates the study of the spatial progression throughout chromatin domains thanks to the simultaneous visualization of all four replication time points. Like the Heisenberg uncertainty principle [19], achieving the highest possible spatial resolution is often incompatible with acquiring the most detailed temporal information. 
2 Materials

\subsection{Live-Cell Visualization of DNA Replication Dynamics}

All solutions and materials used for cell culture and live-cell microscopy must be sterile.

1. Cell lines: for live-cell microscopy, cells should grow adherently. While the cell line to be used depends on the interest of the scientist, there are certain considerations simplifying the acquisition of data (see Note 1). In general, transiently expressed fluorescently tagged replication factors are necessary. However, there are also stable cell lines available [15].

2. Growing medium: use the standard medium required for the cell line to be imaged.

3. Pre-warmed PBS containing $0.5 \mathrm{mM}$ EDTA and $0.05 \%$ trypsin.

4. Plasmids: mammalian expression vectors coding for the replication factor of interest tagged to a fluorescent protein. The fluorescent marker should be chosen according to the wavelengths that can be imaged using the microscope available. It is possible to combine different fluorescent markers, also depending on the microscope setup. The most standard marker, which can typically be imaged in most microscopes, is green fluorescent protein (GFP) [20]. Replication factors most commonly used to label sites of ongoing replication in living cells are PCNA and DNA Ligase 1 [14, 15, 21].

5. Transfection reagents: nucleofection system from Amaxa (Lonza), nucleofection solution V, cuvette, and pipette (see Note 2).

6. Microscopy dishes: the form and size depends on the optical table inset available in the microscope. The bottom has to be thin enough for higher magnification objectives to be able to image through to the sample. Material can be glass or optical plastic. Glass lids are recommended for optimal contrast images ( see Note 3).

7. Microscope: for high-resolution imaging of living cells, we recommend the use of a spinning disk confocal microscope, characterized by high-speed acquisition and low phototoxicity to cells. The stage should be motorized to allow the acquisition of 3D stacks at several points in one experiment.

8. Incubation chamber: the incubation chamber on the microscope must keep a constant temperature, $\mathrm{CO}_{2}$, and humidity imitating the normal cell growth conditions ( see Note 4).

\subsection{Polytemporal DNA Replication Staining}

1. Growth medium.

Human cervical cancer cells (HeLa) and M. cabrerae fibroblasts [22] are cultivated in Dulbecco's Modified Eagle's Medium (DMEM) supplemented with $10 \%$ fetal calf serum (FCS), $2 \mathrm{mM}$ L-glutamine, and $25 \mathrm{mg} / 1$ gentamicin. 
2. Denaturation.

(a) Enzymatic denaturation: DNaseI (Roche), $2 \times$ DNase buffer: mix $60 \mathrm{mM}$ Tris- $\mathrm{HCl} \mathrm{pH}$ 8.1, $0.66 \mathrm{mM} \mathrm{MgCl}_{2}$ and $\mathrm{l} \mathrm{mM}$ mercaptoethanol in $\mathrm{ddH}_{2} \mathrm{O}$.

(b) Acid denaturation: mix $336 \mu \mathrm{l} 12 \mathrm{~N} \mathrm{HCl}$ with $10 \mu \mathrm{l}$ Triton X-100 and $654 \mu \mathrm{lddH_{2 }} \mathrm{O}$ (freshly prepared).

3. PBST wash buffer.

Mix $1 \times$ PBS with Tween 20 to a final concentration of $0.01 \%$.

4. PBSTE DNAse stop buffer.

Add $1 \mathrm{mM}$ EDTA to $1 \times$ PBST.

5. Blocking buffers.

$4 \%$ bovine serum albumin (BSA) in PBS.

$0.2 \%$ fish skin gelatin (Sigma) in PBS.

6. Primary antibodies/chemical detection.

(a) Click-iT EdU Alexa Fluor 488 Imaging Kit (Invitrogen).

(b) Rat anti-bromodeoxyuridine (BrdU), reacts weakly with chlorodeoxyuridine (CldU) (Gentaur Molecular Products, catalog no. OBT0030CX).

(c) Mouse anti-bromodeoxyuridine (BrdU), reacts also with iododeoxyuridine (IdU) (Becton Dickinson, catalog no. 347580).

(d) Anti-DNA Ligase 1 rabbit polyclonal antibody [14].

7. Secondary antibodies (see Note $\mathbf{5}$ ).

(a) CF405M-conjugated goat anti-mouse $\operatorname{IgG}(\mathrm{H}+\mathrm{L})$ highly cross-adsorbed, $2 \mathrm{mg} / \mathrm{mL}$ (Biotium).

(b) Cy5-conjugated AffiniPure donkey anti-rat IgG $(\mathrm{H}+\mathrm{L})$ highly cross-adsorbed, $\quad 1.5 \mathrm{mg} / \mathrm{mL}$ (Jackson ImmunoResearch Europe).

(c) Cy3-conjugated AffiniPure donkey anti-rabbit $\operatorname{IgG}(\mathrm{H}+\mathrm{L})$ highly cross-adsorbed, $\quad 1.5 \mathrm{mg} / \mathrm{mL}$ (Jackson ImmunoResearch Europe).

8. Mounting.

Add $8 \mathrm{~g}$ Moviol 4-88 (Polyscience) to $40 \mathrm{ml} 0.2 \mathrm{M}$ Tris- $\mathrm{HCl}$ $\mathrm{pH} 8.5$ and dissolve by heating to $50-60{ }^{\circ} \mathrm{C}$ with occasional stirring. After cooling down, add $20 \mathrm{ml}$ glycerol and $\mathrm{l}-2.5 \%$ DABCO (anti-fading agent) and spin at $3,800 \times g$ for $15 \mathrm{~min}$, aliquot supernatant and store at $-20{ }^{\circ} \mathrm{C}$.

Alternatively, use Vectashield mounting medium (Vector Laboratories, Inc.). 


\section{Methods}

Below, we provide detailed protocols for (1) replication labeling by live-cell microscopy for mouse embryonic fibroblasts and (2) fixed cell polytemporal replication imaging in human cervical cancer cells and cabrera's vole fibroblasts, a rodent species endemic from the Iberian peninsula [22].

\subsection{Live-Cell}

Visualization of DNA Replication Dynamics
Here, we present a detailed protocol to image active sites of DNA replication and, simultaneously, highlight specific nuclear regions, in this case using MaSat-GFP, a polydactyl zinc finger protein that specifically binds the major satellite repeats enriched at mouse constitutive heterochromatin (see Fig. 1 and Movie S1).
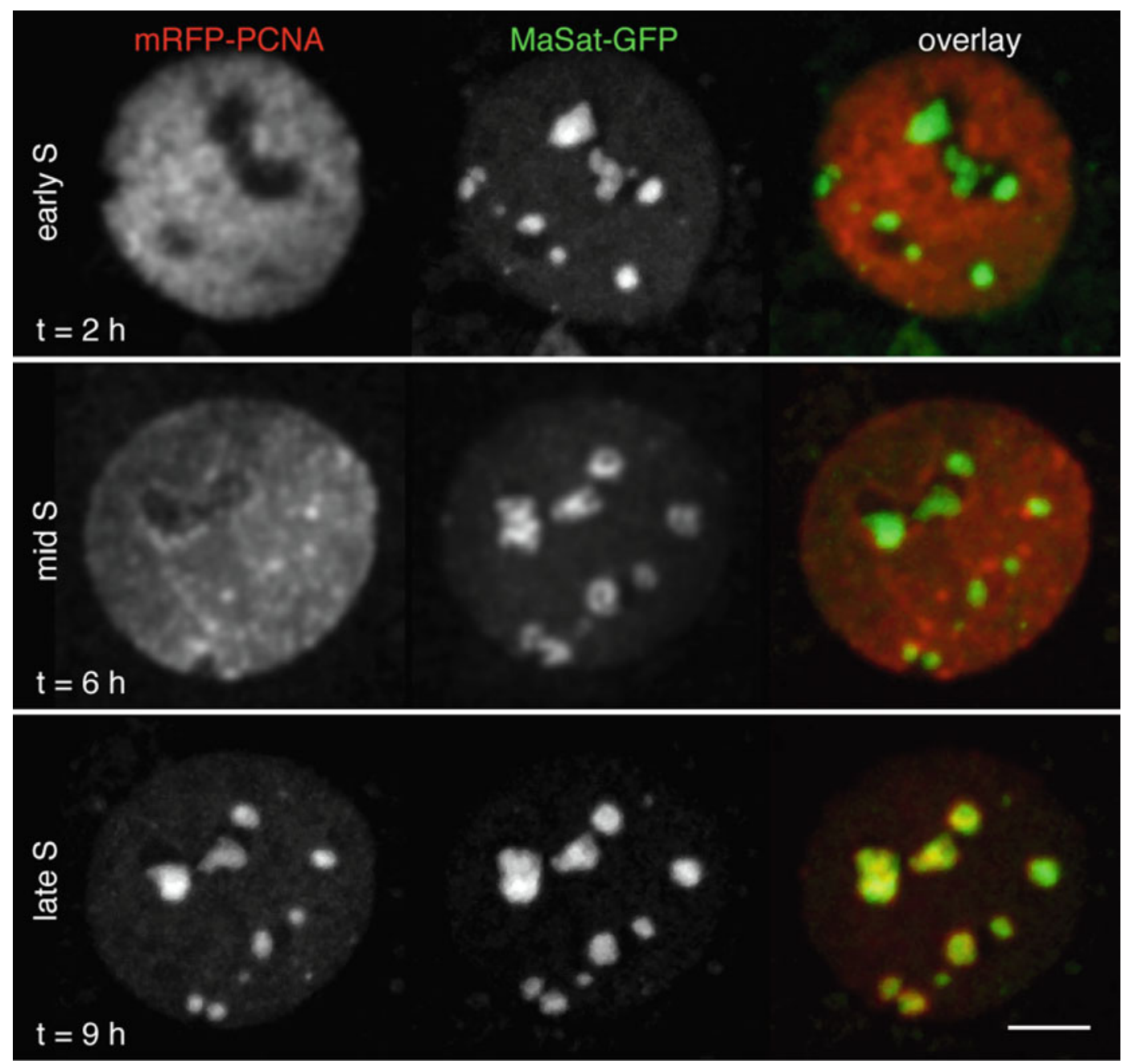

Fig. 1 Mouse embryonic fibroblast transiently co-expressing mRFP-PCNA, as a marker for sites of ongoing DNA replication and MaSat-GFP, as a marker for heterochromatin. Cells were imaged in a spinning disk microscope equipped with a climatization chamber so as to maintain constant temperature $\left(37^{\circ} \mathrm{C}\right), 5 \% \mathrm{CO}_{2}$ and $60 \%$ humidity. 3D confocal stacks were acquired at $1 \mathrm{~h}$ time intervals for a period of $12 \mathrm{~h}$. Exemplary images of the same cell undergoing different S-phase stages and exhibiting the corresponding characteristic patterns. Scale bar: $5 \mu \mathrm{m}$ 
Different nuclear regions can be visualized specifically using various in vivo markers [23-25]. The protocol is adapted to image cells in a $35 \mathrm{~mm}$ glass-bottomed dish.

1. Pre-warm growing medium and PBS +EDTA to $37^{\circ} \mathrm{C}$ and trypsin and nucleofection solution to room temperature. Prepare the dish where electroporated cells will be seeded by adding the final volume of growing medium $(2 \mathrm{ml}$ for a $35 \mathrm{~mm}$ dish) and keep it in an incubator so that the medium reaches $37^{\circ} \mathrm{C}$ and $\mathrm{CO}_{2}$ diffuses into it.

2. Use a $0.5-1 \times 10^{6}$ adherently growing cells (see Note 6) on, e.g., a $10 \mathrm{~cm}$ diameter plate. Remove growing medium and wash carefully with $5 \mathrm{ml}$ PBS EDTA so as not to detach cells from the surface. Add $0.5 \mathrm{ml}$ trypsin and incubate at $37^{\circ} \mathrm{C}$ for 2-5 min. Monitor cell detachment under a microscope. When most cells have detached from the growing substrate and are now single cells, stop the reaction by addition of $4.5 \mathrm{ml}$ growing medium. If cells clump, carefully pipette the cell suspension up and down a couple of times before stopping the trypsin reaction. Centrifuge the cells for $7 \mathrm{~min}$ at $300 \times \mathrm{g}$.

3. Prepare $100 \mu \mathrm{l}$ of nucleofection solution with $2 \mu \mathrm{g}$ total plasmid DNA.

4. Once the cells are pelleted, discard the supernatant and carefully resuspend them in $100 \mu \mathrm{l}$ nucleofection solution with plasmid DNA. Transfer the cell suspension into an appropriate cuvette avoiding air bubbles. Immediately perform the electroporation using the appropriate program for your cells (see Note 7). Take the previously prepared dish from the incubator, pipette approximately $500 \mu \mathrm{l}$ medium from the dish into the cuvette, and carefully resuspend the cells. Transfer cell suspension into the dish with the rest of the medium; carefully shake the dish and return it to the incubator. Incubate overnight.

5. On the next day, remove the medium, carefully wash once or twice with pre-warmed medium to remove dead cells and debris, and add new medium.

6. Before bringing the cells to the microscope, make sure that the incubation chamber is already at $37^{\circ} \mathrm{C}, 5 \% \mathrm{CO}_{2}$, and $>40 \%$ humidity level.

7. Place the dish with the transfected cells on the microscope. Allow the dish to acclimatize to the new conditions for some minutes before starting imaging. Slight changes in temperature can affect the material in such a way that the focal plane can change dramatically during the first 10-20 min.

8. Look for transfected cells using the longest wavelength possible and short exposure times to minimize phototoxicity (i.e., in the case of co-expression of GFP and mRFP tagged proteins, 
look for transfected cells using the red channel and only quickly check to see whether the cells also express the GFP-tagged construct). Select cells (see Note 8) that express the minimal amount of fluorescent protein that can be imaged properly. Too high expression levels can lower the chances that transfected cells will pass normally through S-phase. Extreme overexpression can also cause apoptosis. In case the lid is to be removed/replaced, do so before selecting the cells for imaging, since the dish might otherwise be shifted (see Note 9).

9. Set up the imaging conditions finding a compromise between phototoxicity and undersampling. The ideal conditions depend strongly on the cell line, since some cells are more sensitive to transfection and phototoxicity. In general, acquiring $z$-stacks at a time interval of $10-30 \mathrm{~min}$ is usually enough to follow changes in replication patterns. The minimal time to acquire an entire S-phase/cell cycle depends on how fast the cells divide. Under optimal conditions, cells can be kept on the stage and imaged for over 2 days (see Note 10).

3.2 Polytemporal DNA Replication Staining
Here, we present the detailed protocol for imaging multiple replication time points in mammalian cells (see Fig. 2). Proliferating cells are supplied with thymidine analogs, which are incorporated in the replicating genomic DNA for the duration of the pulse. Incorporation of modified nucleotides is stopped by chasing with excess unlabeled thymidine ( see Note 11). The protocol is adapted for multiple $16 \mathrm{~mm}$ diameter coverslips in a $60 \mathrm{~mm}$ cell culture dish. Pulse-chase length needs to be adapted to doubling time and $S$-phase duration of the respective cell line, as well as to the purpose of the experiment. In principle, any replicating, cultured cells can be used for this approach.

1. Replication pulse labeling with thymidine analogs (IdU, EdU, and $\mathrm{CldU}$ ).

Label, e.g., $20 \mathrm{~min}$ with a final concentration of $10 \mu \mathrm{M}$ IdU in growth medium at $37^{\circ} \mathrm{C}$, rinse twice with pre-warmed growth medium, and incubate cells for, e.g., $60 \mathrm{~min}$ with $200 \mu \mathrm{M}$ thymidine in growth medium at $37^{\circ} \mathrm{C}$. Rinse again twice to remove thymidine from growth medium.

Label 20 min with a final concentration of $10 \mu \mathrm{M} \mathrm{EdU}$ in growth medium at $37^{\circ} \mathrm{C}$, rinse two times with growth medium, and incubate cells for $60 \mathrm{~min}$ with $200 \mu \mathrm{M}$ thymidine in growth medium at $37^{\circ} \mathrm{C}$. Rinse again twice to remove thymidine from growth medium.

Label 20 min with a final concentration of $10 \mu \mathrm{M} \mathrm{CldU}$ in growth medium at $37^{\circ} \mathrm{C}$, rinse two times with growth medium, and incubate cells for $60 \mathrm{~min}$ with $200 \mu \mathrm{M}$ thymidine in growth medium at $37^{\circ} \mathrm{C}$ ( see Note 12). 

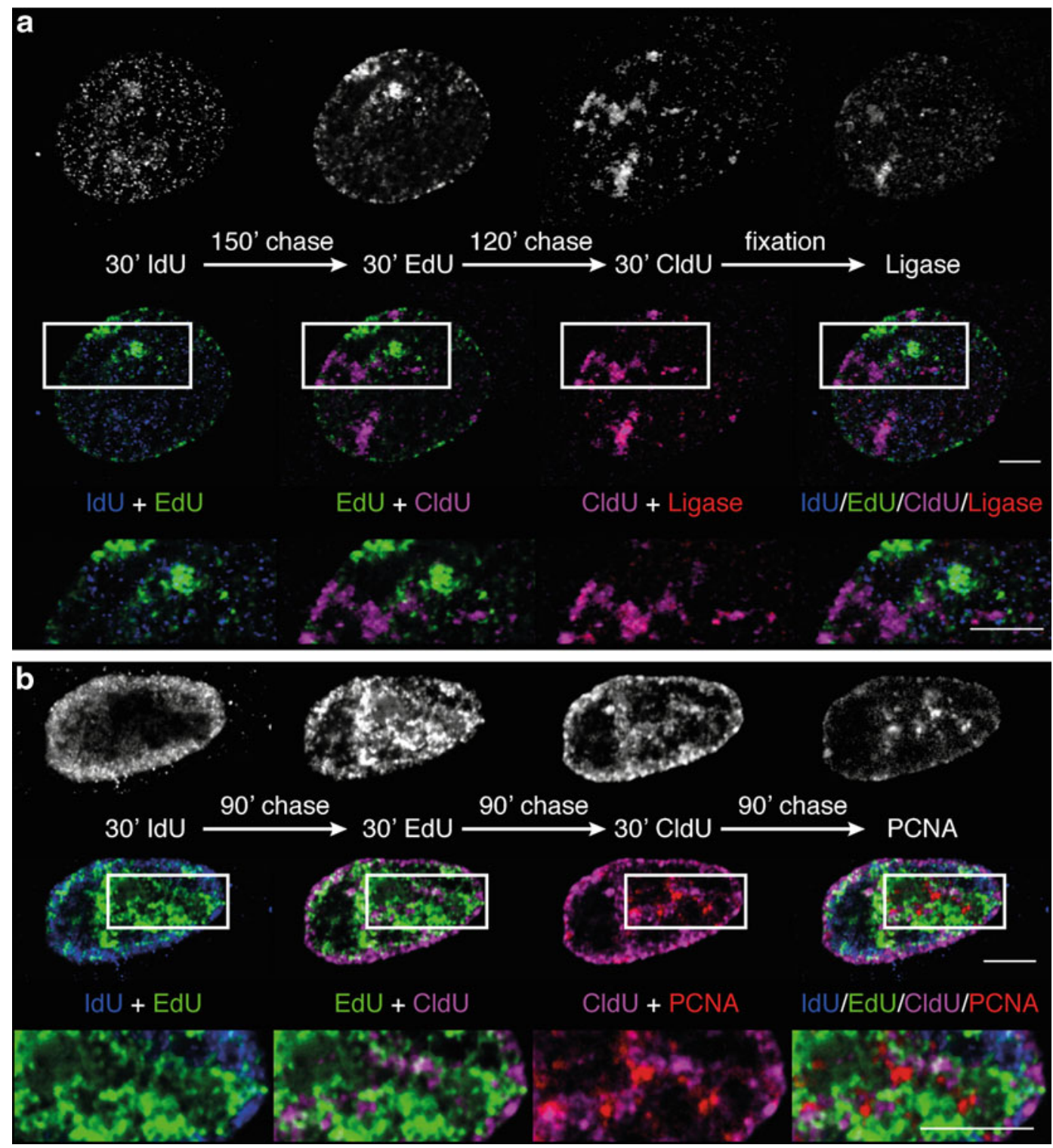

Fig. 2 Quadruple replication labeling in mammalian cells. Mid optical section of confocal images. (a) Six hour multiple pulse-chase replication staining in M. cabrerae fibroblast with immediate fixation after the CldU pulse. IdU pulse in blue, EdU pulse in green, CldU pulse in magenta, and DNA Ligase 1 in red, pulse and chase times as given in the image. (b) Six hour multiple pulse-chase replication staining in HeLa cells expressing mCherryPCNA with fixation following CldU pulse and chase. IdU pulse in blue, EdU pulse in green, CldU pulse in magenta, and PCNA in red, pulse and chase times as given in the image. Top row. single replication stainings. Middle row. overlaid images of two sequential pulses followed by an overlay of all four pulses. Bottom row. selected ROI from the middle row. Scale bars: $5 \mu \mathrm{m}$ 
2. Fixation.

Rinse cells once with PBS at room temperature to remove growth medium before fixing with $3.7 \%$ formaldehyde in PBS for $10 \mathrm{~min}$.

Remove fixative and rinse two to three times with PBST.

Cells can be stored at this point in PBS with $0.02 \%$ Na-azide at $4{ }^{\circ} \mathrm{C}$ for several days.

3. Staining chamber.

Prepare a lightproof chamber with a wet filter paper to maintain a humid environment and place parafilm on top of the filter [26]. Transfer coverslips to the chamber (see Note 13).

4. Permeabilization.

To allow antibody penetration, permeabilize the cells with $0.5 \%$ Triton X-100 in PBS for 10-20 min at room temperature. Wash the cells three times in PBST for 0,5 , and $10 \mathrm{~min}$ (hereafter referred to as triple wash) to remove the permeabilization reagent (see Note 14).

5. Detection of replication labeling ( see Note 15).

Use the ClickiT-EdU kit according to manufacturer's protocol to detect incorporated EdU followed by a triple wash (see Note 16).

Block with $0.2 \%$ fish skin in PBS for $45 \mathrm{~min}$ at room temperature.

Mix $0.25 \mu \mathrm{l}$ rat anti-BrdU Gentaur antibody solution (CldU detection) with $12.5 \mu \mathrm{l} 2 \times$ DNase buffer and $12.5 \mu \mathrm{l} 4 \%$ BSA in PBS. Add $1 \mathrm{U}$ DNaseI and incubate at $37^{\circ} \mathrm{C}$ for $1 \mathrm{~h}$, followed by a triple wash with PBSTE (see Notes 17 and 18).

Mix $6.25 \mu \mathrm{l}$ mouse anti-BrdU Becton Dickinson antibody solution (IdU detection) with $0.25 \mu \mathrm{l}$ rabbit anti-DNA Ligase 1 antibody and $23.5 \mu \mathrm{l} 4 \% \mathrm{BSA}$ in PBS and incubate at room temperature for $60 \mathrm{~min}$, followed by a triple wash.

Mix $0.06 \mu \mathrm{l}$ anti-mouse IgG CF405M, $0.3 \mu \mathrm{l}$ anti-rat IgG Cy5, $0.5 \mu \mathrm{l}$ anti-rabbit IgG Cy3, and $24 \mu \mathrm{l} 4 \% \mathrm{BSA}$ in PBS to perform secondary antibody incubation at room temperature for $60 \mathrm{~min}$, followed by a triple wash.

6. Mounting.

Rinse with $\mathrm{ddH}_{2} \mathrm{O}$ to remove salts. Mount coverslips on microscope glass slide with Vectashield and seal with nail polish or mount with Moviol (see Note 19).

\section{Notes}

1. Factors to consider when choosing a cell line to perform livecell microscopy include:

- How well the cells can be transfected (transfection and expression rate)

- How well the cells tolerate imaging-derived phototoxicity 
- How much the cells move

- How fast they divide

2. While a good transfection rate is a factor to consider when choosing a transfection method, when following cell cycle progression at a single cell level, it is more important to achieve a moderate expression level. We recommend the nucleofection system from Amaxa (Lonza), although other methods can also be used.

3. Usually, the lid of microscopy dishes is of a thick plastic, which prevents good contrast images. For short-term imaging, it is possible to simply remove the lid.

4. If it is not possible to regulate the $\mathrm{CO}_{2}$ and humidity levels, an alternative is to add $10 \mathrm{mM}$ HEPES buffer to phenol-free medium and seal the dish with, for instance, paraffin to avoid evaporation of the medium.

5. Secondary antibodies indicated here can be substituted with your own favorite antibodies, as long as they are cross-absorbed to avoid cross-reaction with the other antibodies and the fluorophores conjugated provide enough spectral separation to be imaged by your microscope.

6. Cell density is a key factor for live-cell imaging of DNA replication. While a too high density can result in cell contact inhibition and therefore prevent cells from cycling, a too low density can result in cells moving more freely along the growing surface, making it extremely hard to keep them in frame over several hours. The optimal cell density depends on the cell line used: mouse fibroblasts and myoblasts tend to move, and therefore, a rather high density is recommended. On the other hand, HeLa cells, for instance, are less mobile and can therefore be plated at a lower density.

7. The program B-032 gives good results for mouse embryonic fibroblasts, $\mathrm{C} 2 \mathrm{Cl} 2$ mouse myoblasts, HeLa cells, and $M$. cabrerae fibroblasts.

8. If the acquisition software allows the possibility of multipoint and mosaic imaging (stitching), the decision should be made depending on both the transfection rate and the mobility of the cell line used: a high transfection rate and cells that move fast are better imaged using the mosaic function, while sparsely transfected cells, or cells that barely move, are better imaged selecting separate points across the dish.

9. For time-lapse imaging over several hours, removing the lid would result in evaporation of the medium and possibly contamination of the sample. Therefore, in such cases it is better to replace the lid by a glass coverslip or keep the plastic lid.

10. In an asynchronous population at any given time point, the user will observe cells undergoing all of the different cell cycle substages. 
Mitotic cells are easily recognized using phase contrast or DIC by their spherical morphology. At this stage of the cell cycle, PCNA diffuses throughout the cytoplasm. During Gl and G2, PCNA is exclusively nuclear but distributed homogenously. As S-phase begins, PCNA distribution becomes focal. During early S-phase the replication foci labeled by PCNA are distributed throughout the nucleus with the exception of the nuclear and nucleolar periphery. As cells progress into mid $S$-phase, the replication foci relocalize to these peripheral regions. During the second half, or late, S-phase, replication foci cluster at heterochromatic regions, giving the impression, at the confocal microscopy level, of fewer but larger foci (Fig. I and Movie S1).

11. With this pulse-chase-pulse labeling protocol, sequentially replicated regions of the genome are marked with differently modified nucleotides and visualized simultaneously within single cells. This protocol allows following the spatiotemporal dynamics of DNA replication progression in situ (Fig. 2).

12. We chose this specific chronological labeling order to facilitate visualization of potential cross-reaction between IdU and CldU antibodies. The additional intermediate EdU labeling time point allows a clear discrimination between both IdU and CldU labeling patterns. A high colocalization between both IdU and CldU antibodies signals indicates spurious cross-reaction of the primary antibodies with both nucleotides.

13. To reduce the required amount of antibodies, we use a "cell down protocol." A $25 \mu \mathrm{l}$ drop of staining reagent for each $16 \mathrm{~mm}$ diameter coverslip was placed on the parafilm in the humidified chamber. The coverslips were placed with cells facing downward onto the reagent carefully avoiding any air bubbles. If conventional methods are used instead of the cell down protocol, the respective amounts of reagents have to be adapted.

14. For PCNA detection, incubate cells for $10 \mathrm{~min}$ in ice-cold methanol and air-dry coverslips. Cell shape is usually affected by $\mathrm{MeOH}$ incubation and air-drying.

15. Nucleotide labeling and fluorescently coupled proteins are readily combinable with other (immuno) fluorescent labeling methods. Chromosome painting, methylation labeling, and chromocenter labeling are only a few among many possible permutations.

16. We observed intensity decrease in other fluorochromes when the ClickiT-EdU kit was applied as the final detection step.

17. Primary antibodies rat anti-BrdU Gentaur and mouse anti-BrdU Becton Dickinson were detected sequentially to minimize crossreaction. A high salt wash in between is not essential but improves the quality of the staining by decreasing cross-reactions. 
18. Alternatively to DNaseI treatment, acid denaturation can be performed after permeabilization and before the first detection step to expose the modified nucleotides for antibody recognition. For acid denaturation, incubate with $4 \mathrm{~N} \mathrm{HCl}$ solution (see Subheading 2) for $10 \mathrm{~min}$ at room temperature. While acid denaturation can cause artifacts and partially damage protein epitopes, such as the ones recognized by the anti-DNA Ligase 1 antibodies, nucleotide recognition is generally improved.

19. Each mounting agent has different advantages. Vectashield allows later unmounting and restaining, whereas Moviol is a permanent mounting agent.

\section{Acknowledgements}

We thank Juan Alberto Marchal (University of Jaen, Spain) for the Microtus cabrerae fibroblasts and all present and past members of the laboratory for their contributions over the years. The laboratory of M. Cristina Cardoso is supported by grants of the German Research Foundation (DFG) and the Federal Ministry of Education and Research (BMBF).

\section{References}

1. Berezney R, Dubey DD, Huberman JA (2000) Heterogeneity of eukaryotic replicons, replicon clusters, and replication foci. Chromosoma 108:471-484

2. Nakamura H, Morita T, Sato C (1986) Structural organizations of replicon domains during DNA synthetic phase in the mammalian nucleus. Exp Cell Res 165:291-297

3. Nakayasu H, Berezney R (1989) Mapping replicational sites in the eucaryotic cell nucleus. J Cell Biol 108:1-11

4. O'Keefe RT, Henderson SC, Spector DL (1992) Dynamic organization of DNA replication in mammalian cell nuclei: spatially and temporally defined replication of chromosomespecific alpha-satellite DNA sequences. J Cell Biol 116:1095-1110

5. Huberman JA, Riggs AD (1966) Autoradiography of chromosomal DNA fibers from Chinese hamster cells. Proc Natl Acad Sci USA 55:599-606

6. Huberman JA, Tsai A, Deich RA (1973) DNA replication sites within nuclei of mammalian cells. Nature 241:32-36

7. Gratzner HG (1982) Monoclonal antibody to 5-bromo- and 5-iododeoxyuridine: a new reagent for detection of DNA replication. Science 218:474-475
8. Bravo R, Macdonald-Bravo H (1985) Changes in the nuclear distribution of cyclin (PCNA) but not its synthesis depend on DNA replication. EMBO J 4:655-661

9. Cardoso MC, Leonhardt H, Nadal-Ginard B (1993) Reversal of terminal differentiation and control of DNA replication: cyclin A and Cdk2 specifically localize at subnuclear sites of DNA replication. Cell 74:979-992

10. Aten JA, Bakker PJ, Stap J, Boschman GA, Veenhof CH (1992) DNA double labelling with IdUrd and CldUrd for spatial and temporal analysis of cell proliferation and DNA replication. Histochem J 24:251-259

11. Manders E, Stap J, Brakenhoff G, Van Driel R, Aten J (1992) Dynamics of three-dimensional replication patterns during the S-phase, analysed by double labelling of DNA and confocal microscopy. J Cell Sci 103:857-862

12. Schermelleh L, Solovei I, Zink D, Cremer T (2001) Two-color fluorescence labeling of early and mid-to-late replicating chromatin in living cells. Chromosome Res 9:77-80

13. Chalfie M, Tu Y, Euskirchen G, Ward WW, Prasher DC (1994) Green fluorescent protein as a marker for gene expression. Science 263:802-805

14. Cardoso MC, Joseph C, Rahn HP, Reusch R, Nadal-Ginard B, Leonhardt H (1997) Mapping 
and use of a sequence that targets DNA ligase I to sites of DNA replication in vivo. J Cell Biol 139:579-587

15. Leonhardt H, Rahn H, Weinzierl P, Sporbert A, Cremer T, Zink D, Cardoso M (2000) Dynamics of DNA replication factories in living cells. J Cell Biol 149:271-280

16. Sporbert A, Gahl A, Ankerhold R, Leonhardt $\mathrm{H}$, Cardoso MC (2002) DNA polymerase clamp shows little turnover at established replication sites but sequential de novo assembly at adjacent origin clusters. Mol Cell 10:1355-1365

17. Chagin VO, Stear JH, Cardoso MC (2010) Organization of DNA Replication. Cold Spring Harb Perspect Biol 2

18. Casas-Delucchi CS, Brero A, Rahn H-P, Solovei I, Wutz A, Cremer T, Leonhardt H, Cardoso MC (2011) Histone acetylation controls the inactive $\mathrm{X}$ chromosome replication dynamics. Nat commun 2:222

19. Heisenberg W (1927) Über den anschaulichen Inhalt der quantentheoretischen Kinematik und Mechanik. Zeitschrift für Physik 43:172-198

20. Shaner NC, Steinbach PA, Tsien RY (2005) A guide to choosing fluorescent proteins. Nat Methods 2:905-909

21. Sporbert A, Domaing P, Leonhardt H, Cardoso MC (2005) PCNA acts as a stationary loading platform for transiently interacting Okazaki fragment maturation proteins. Nucleic Acids Res 33:3521-3528

22. Bullejos M, Burgos M, Jimenez R, Sanchez A (1996) Distribution of sister-chromatid exchanges in different types of chromatin in the $\mathrm{X}$-chromosome of Microtus cabrerae. Experientia 52:511-515

23. Lindhout BI, Fransz P, Tessadori F, Meckel T, Hooykaas PJJ, van der Zaal BJ (2007) Live cell imaging of repetitive DNA sequences via GFPtagged polydactyl zinc finger proteins. Nucleic Acids Res 35:e107

24. Casas-Delucchi CS, van Bemmel JG, Haase S, Herce HD, Nowak D, Meilinger D, Stear JH, Leonhardt H, Cardoso MC (2012) Histone hypoacetylation is required to maintain late replication timing of constitutive heterochromatin. Nucleic Acids Res 40:159-169

25. Weidtkamp-Peters S, Rahn H-P, Cardoso MC, Hemmerich P (2006) Replication of centromeric heterochromatin in mouse fibroblasts takes place in early, middle, and late $S$ phase. Histochem Cell Biol 125:91-102

26. Cardoso MC, Leonhardt H (1995). Immunofluorescence techniques in cell cycle studies. In: Pagano, M (ed) Cell cycle: materials and methods. Heidelberg, Springer-Verlag, pp 15-28 